\title{
EMG-Derived Respiration Signal using the Fixed Sample Entropy during an Inspiratory Load Protocol
}

\author{
Luis Estrada, Member, IEEE, Abel Torres, Member, IEEE, Leonardo Sarlabous, and \\ Raimon Jané, Senior Member, IEEE
}

\begin{abstract}
Extracting clinical information from one single measurement represents a step forward in the assessment of the respiratory muscle function. This attracting idea entails the reduction of the instrumentation and fosters to develop new medical integrated technologies. We present the use of the fixed sample entropy (fSampEn) as a more direct method to noninvasively derive the breathing activity from the diaphragm electromyographic (EMGdi) signal, and thus to extract the respiratory rate, an important vital sign which is cumbersome and time-consuming to be measured by clinicians. fSampEn is a method to evaluate the EMGdi activity that is less sensitive to the cardiac activity (ECG) and its application has proven to be useful to evaluate the load of the respiratory muscles. The behavior of the proposed method was tested in signals from two subjects that performed an inspiratory load protocol, which consists of increments in the inspiratory mouth pressure $\left(P_{\text {mouth }}\right)$. Two respiratory signals were derived and compared to the $P_{\text {mouth }}$ signal: the ECG-derived respiration (EDR) signal from the lead-I configuration, and the EMG-derived respiration (EMGDR) signal by applying the fSampEn method over the EMGdi signal. The similitude and the lag between signals were calculated through the cross-correlation between each derived respiratory signal and the $P_{\text {mouth }}$. The EMGDR signal showed higher correlation and lower lag values $(\geq 0.91$ and $\leq 0.70 \mathrm{~s}$, respectively) than the EDR signal ( $\geq 0.83$ and $\leq$ $0.99 \mathrm{~s}$, respectively). Additionally, the respiratory rate was estimated with the $P_{\text {mouth }}$, EDR and EMGDR signals showing very similar values. The results from this preliminary work suggest that the fSampEn method can be used to derive the respiration waveform from the respiratory muscle electrical activity.
\end{abstract}

\section{INTRODUCTION}

The respiratory activity provides valuable information for the diagnosis and treatment of patients with sleep-disordered breathing (SBD) [1] and chronic obstructive pulmonary diseases (COPD) [2]. A wide range of signals such as nasal

The first author was supported by the Instituto para la Formación y Aprovechamiento de Recursos Humanos and the Secretaría Nacional de Ciencia, Tecnología e Innovación (IFARHU-SENACYT Program) from the Panama Government under grant 270-2012-273. This work was supported in part by the Secretaria d'Universitats i Recerca del Departament d'Economia i Coneixement de la Generalitat de Catalunya (Consolidated research group GRC: 2014 SGR 1569).

L. Estrada, A. Torres, and R. Jané are with the Universitat Politècnica de Catalunya (UPC), the Institut de Bioenginyeria de Catalunya (IBEC) and the Biomedical Research Networking Center in Bioengineering, Biomaterials and Nanomedicine (CIBER-BBN), c/. Baldiri Reixac 4, 08028, Barcelona, Spain. (e-mail: lestrada@ibecbarcelona.eu,abel.torres@upc.edu, rjane@ibecbarcelona.eu).

A. Torres is also with the Escola Universitària d'Enginyeria Tècnica Industrial de Barcelona (EUETIB), Barcelona, Spain.

L. Sarlabous is with the CIBER-BBN and the IBEC (email:Isarlabous@ibecbarcelona.eu). airflow, mouth airflow, inspiratory mouth pressure, respiratory inductance plethysmography or signals recorded with accelerometers have been used for the measurement of the breathing activity with the intention to extract clinical information. On the other hand, there is an interest to use reduced and low-cost instrumentation with the added benefit of extracting various clinical features from one single measurement [3]-[5]. The electrocardiographic (ECG) signal contains not only information related to the cardiac activity but also to the respiration [4], [6]. Respiration can be derived and reconstructed from the ECG signal using methods that exploit the fact that the breathing induces changes in the ECG signal. As we breathe the transthoracic impedance (filling and emptying of the lungs), and the electrode movement relative to the heart induce a modulation over the recorded ECG signal [4]. However, the performance of this method can be limited when the ECG signal is contaminated with artifacts, or with the presence of variations on the ECG morphology or of ectopic beats.

On the other hand, there are other alternatives that allow extracting the breathing activity from a single measurement. During the recording of the ECG, the electromyographic (EMG) activity is also registered. The inspiratory muscles are activated in the inspiratory phase and therefore the EMG signal can be non-invasively detected on the ECG derivations. The EMG activity has been extracted by highpass filtering the ECG signal $(\sim 250 \mathrm{~Hz})$ acquired in magnetic resonance imaging studies [7]. However, the authors reported that the ECG signal is distorted due to the electromagnetic interference of the magnetic field introduced during the magnetic resonance imaging acquisitions, which reduce the performance of deriving the respiration from the EMG signal. In other approach, the respiratory activity was monitored in neonates in intensive care units by deriving the diaphragm electromyographic (EMGdi) signal and masking the ECG signal using a system based on a digital processor [8].

Although the mentioned techniques are useful for the extraction of the EMG activity and therefore permit to derive the breathing activity, they have the inherent problem of having to deal with the ECG contamination. To overcome this shortcoming, in this work, we propose the use of the fixed sample entropy (fSampEn) [10], [11], a technique based on the sample entropy [9]. The use of the fSampEn has proven to be less sensitive in the presence of cardiac activity in comparison to the average rectified value (ARV) and the root mean square (RMS) parameters [10], [11].

In the clinical context, the EMGdi signal presents valuable information, particularly related to the neural respiratory drive, as an indirect measurement of the load exerted by the respiratory muscles [12]. In this study we investigate the use of the fSampEn to derive the breathing 
activity from the EMGdi signal during an inspiratory load protocol which consists of increments in the inspiratory mouth pressure. First, we evaluate the similitude and the lag between the EMG-derived respiration (EMGDR) signal, the ECG-derived respiration (EDR) signal and the the recorded inspiratory mouth pressure $\left(\mathrm{P}_{\text {mouth }}\right)$. Second, we assess the respiratory rate in the $\mathrm{P}_{\text {mouth, }}$ the EDR and the EMGDR signals and compare them with each other.

\section{MATERIAL AND METHODS}

\section{A. Experimental procedure}

Two healthy nonsmoking men who were free of respiratory diseases took part in this study. The subjects performed an inspiratory load protocol in which they first inhaled via a mouthpiece at quiet breathing. Next, an inspiratory load was imposed using an attached hand-held inspiratory muscle training device (Threshold IMT, Philips Respironics, Amsterdam, The Netherlands). The inspiratory load was set at a level of $9,17,25$ and $33 \mathrm{~cm} \mathrm{H}_{2} \mathrm{O}$. The experimental procedure was performed with the subjects seated and wearing a nose clip to prevent nasal breathing with 2 min of rest between each inspiratory load of $1 \mathrm{~min}$ length. The breathing frequency was regulated with a visual feedback provided from a screen at a rate of 16 breaths per minute and displayed the timing information of the breathing cycles. The study was conducted with the subject's written consent, and with the approval of the Institutional Review Board of the Institute for Bioengineering of Catalonia (IBEC), Barcelona, Spain.

\section{B. Signal acquisition}

The ECG activity was recorded from the standard lead-I configuration using $\mathrm{Ag} / \mathrm{AgCl}$ electrodes (pre-gelled, disposable, 10-mm diameter contact area, foam electrode 50/PK - EL501, Biopac Systems Inc, Santa Barbara, CA, USA), plugged into a modular amplifier (ECG 100C, Biopac Systems Inc) with a gain of 2000 and an analog high-pass filter with a cut-off frequency of $0.5 \mathrm{~Hz}$. Moreover, the EMGdi activity was recorded using two $\mathrm{Ag} / \mathrm{AgCl}$ electrodes in bipolar configuration placed on the lower right chest along the anterior axillary line, over the $7^{\text {th }}$ and $8^{\text {th }}$ intercostal spaces and above the costal margin. The EMGdi signal was amplified using a modular amplifier (EMG 100C, Biopac Systems), with a gain of 5000 and an analog band-pass filter with cut-off frequencies from 1 to $500 \mathrm{~Hz}$. Additionally, an $\mathrm{Ag} / \mathrm{AgCl}$ electrode was placed at the left ankle as a reference. Simultaneously, the inspiratory mouth pressure $\left(\mathrm{P}_{\text {mouth }}\right)$ was measured by a differential pressure transducer (TSD160, Biopac Systems), amplified using a modular differential amplifier (DAC100C, Biopac Systems) with a gain of 50 and an analog low-pass filter with a cut-off frequency of $300 \mathrm{~Hz}$. The signals were digitized at a sampling rate of $2000 \mathrm{~Hz}$ using a data acquisition system (MP150, Biopac Systems Inc), visualized in real time and stored (AcqKnowledge software v.3.2, Biopac Systems Inc). All signals have been subsampled at $1000 \mathrm{~Hz}$. Data analysis was performed using MATLAB (v. R2011b, Natick, MA, USA).

\section{ECG-derived respiration}

The EDR signal was extracted from the lead-I ECG signal based on the methodology described in [5]. This methodology consists of detecting the ECG beats and measuring the peak to peak amplitude of the QRS complex in each beat. The outlier values greater than two standard deviations in non-overlapping windows of $30 \mathrm{~s}$ were rejected (due to noise and artifacts). Next, the EDR signal was generated using cubic-spline interpolation with a frequency of $8 \mathrm{~Hz}$. Finally, as the EDR signal is in antiphase with respect to $\mathrm{P}_{\text {mouth }} \mathrm{f}$ and EMGDR signals, the EDR signal was inverted.

\section{EMG-derived respiration using fSampEn}

The EMGdi signal is affected by artifacts and other bioelectrical signals, being the ECG the main source of noise. Additionally, both the ECG and the EMGdi power spectrum are overlapped and in consequence, it affects the proper evaluation of the respiratory muscle activity. To alleviate the aforementioned problems, we have recently proposed the use of the fixed sample entropy (fSampEn) [11]. fSampEn has the advantage of being less sensitive in the presence of the ECG in comparison to ARV and RMS amplitude based parameters, and therefore with the potential of being used for the evaluation of EMGdi signal and the quantification of the neural respiratory drive [11].

Fig. 1 shows a simulated periodical signal with added impulsive noise and the use of fSampEn, ARV and RMS. It is remarkable that fSampEn has a better performance in comparison to ARV and RMS in the presence of impulsive noise. The fSampEn is a variation of the sample entropy (SampEn) [9]. SampEn makes use of two parameters, the embedding dimension $m$, and the tolerance value $r$. fSampEn is evaluated in a moving window, using fixed $r$ values independent of the standard deviation of each moving window [11]. Furthermore, fSampEn has successfully been used for the evaluation of the diaphragm mechanomyographic activity, the mechanical counterpart of the respiratory muscles [10]. EMGdi signals were evaluated using a moving window of one second in length, with an overlap of $90 \%$. The $m$ and $r$ values were set to 1 and 0.3 times the standard deviation of the EMGdi signal, respectively, based on previous studies [10], [11]. In addition to providing information related to the neural respiratory drive, the use of fSampEn can be used to derive the breathing activity and therefore to extract the respiratory rate. The EMGDR signal was extracted from the EMGdi activity based on the use of fSampEn.

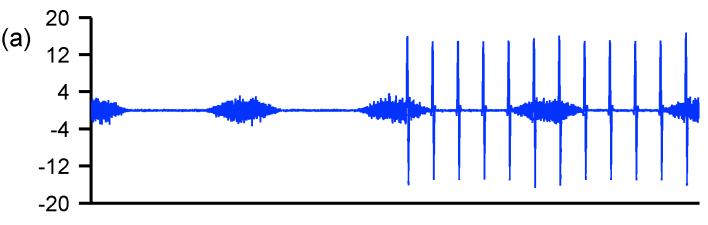

(b)

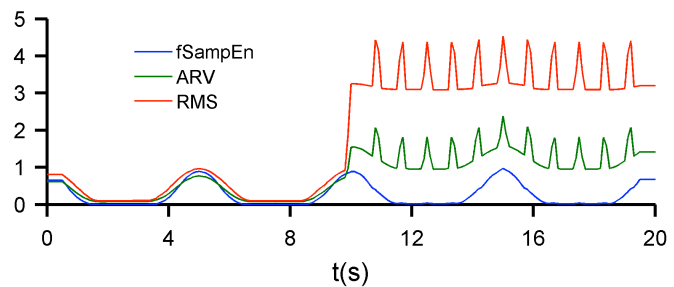

Figure 1. (a) Random signal of varying amplitude with impulsive noise. (b) Application of fSampEn, ARV, and RMS using a moving window of $1 \mathrm{~s}$ with steps of $0.1 \mathrm{~s}$. fSampEn was set to $m=1, r=0.3$ time the standard deviation of the whole signal. 


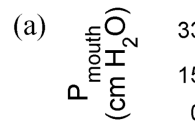

(b) 4

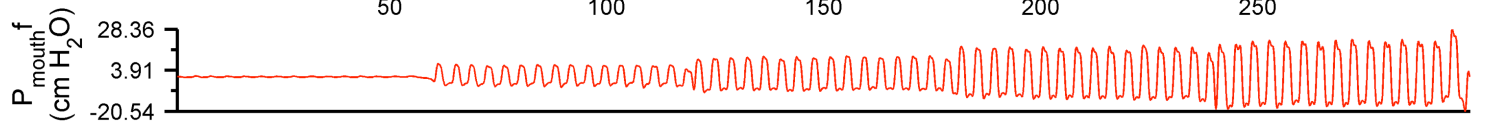

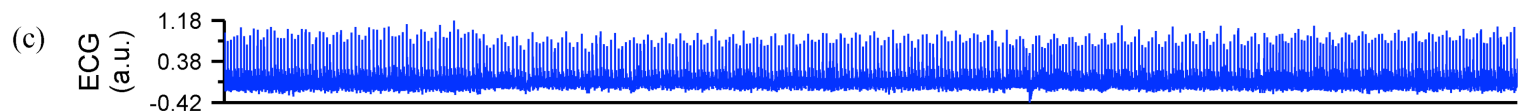

(d)

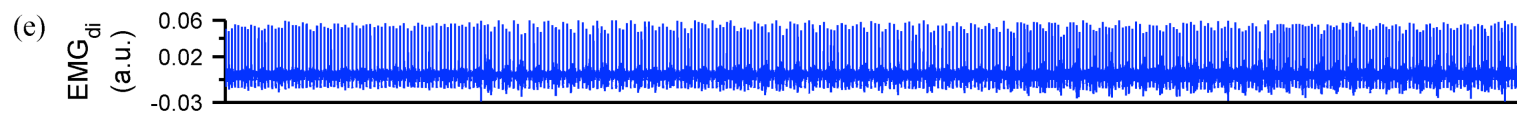

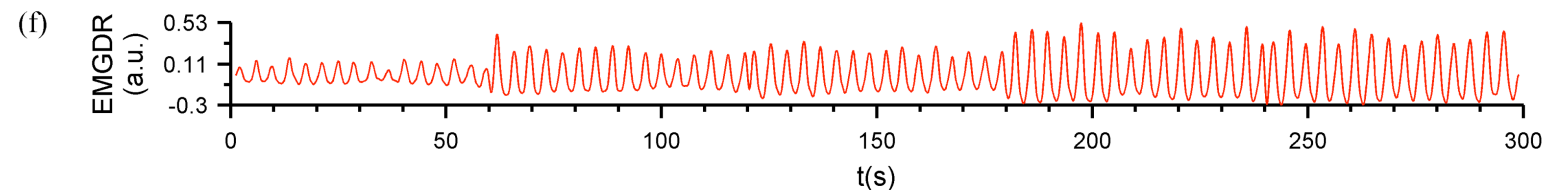

Figure 2. (a) Inspiratory mouth pressure $\left(\mathrm{P}_{\text {mouth }}\right)$, (b) Filtered inspiratory mouth pressure $\left(\mathrm{P}_{\text {mouth }} \mathrm{f}\right),(\mathrm{c})$ ECG-Lead I, $(\mathrm{d})$ ECG-derived respiration $($ EDR $)$ signal from Lead I, (e) Diaphragm electromyographic $\left(\mathrm{EMG}_{\mathrm{di}}\right)$ signal and (f) EMGdi-derived respiration (EMGDR) signal, from a representative subject performing an inspiratory load protocol.

\section{E. Evaluation of the respiratory signals and the respiratory rate}

$\mathrm{P}_{\text {mouth, }}$ EDR and EMGDR signals were resampled at a frequency of $100 \mathrm{~Hz}$ and digitally band-pass filtered using a zero-phase second-order Butterworth filter with a cut frequency of 0.1 and $1 \mathrm{~Hz}$ (corresponding to respiratory rates from 6 to 60 breaths per minute). The filtered $P_{\text {mouth }}$ signal was called $\mathrm{P}_{\text {mouth }} \mathrm{f}$ signal. The cross-correlation was applied to validate the similitude and to calculate the lag between the $\mathrm{P}_{\text {mouth }} \mathrm{f}$ vs EDR, and between the $\mathrm{P}_{\text {mouth }} \mathrm{f}$ vs EMGDR at different levels of the inspiratory load and for the set of all inspiratory loads. Moreover, the respiratory rate was calculated over the $\mathrm{P}_{\text {mouth }} \mathrm{f}$, EDR and EMGDR signals at each level of inspiratory load. With this intention, the power spectral density was estimated using the Welch's modified periodogram method and the peak frequency of the spectrum was calculated.

\section{RESULTS}

Fig. 2 shows an example of the $P_{\text {mouth, the }} P_{\text {mouth }} f$, the ECG, the EDR, the EMGdi and the EMGDR signals evaluated during the incremental inspiratory load protocol. It can be observed that EDR and EMGDR signals represent the breathing movement when compared to the $\mathrm{P}_{\text {mouth }}$ signal. Fig. 3 shows a detail of $10 \mathrm{~s}$ of $\mathrm{P}_{\text {mouth }} \mathrm{f}$, EDR and EMGDR signals at quiet breathing (left column) and at $33 \mathrm{~cm} \mathrm{H}_{2} \mathrm{O}$ (right column). The vertical lines indicate the maximum value in the chosen inspiratory cycle (highlighted by vertical light red rectangles). Both EDR and EMGDR signals are delayed compared to the $\mathrm{P}_{\text {mouth }}$ f signal; the EMGDR signal showed lower delay than the EDR signal.

Table I reported the maximum value of the crosscorrelation and the lags between $\mathrm{P}_{\text {mouth }} \mathrm{f}$ vs EDR and between $\mathrm{P}_{\text {mouth }} \mathrm{f}$ vs EMGDR at different levels of inspiratory load and the set of all inspiratory loads for the two evaluated subjects.

TABLE I. CROSS-CORRELATION AND LAGS AT DIFFERENT LEVELS OF INSPIRATORY LOAD AND THE SET OF ALL INSPIRATORY LOADS

\begin{tabular}{|c|c|c|c|c|c|c|c|c|c|c|c|c|c|}
\hline Subject & & \multicolumn{2}{|c|}{ QB } & \multicolumn{2}{|c|}{ L1 } & \multicolumn{2}{|c|}{$\mathbf{L 2}$} & \multicolumn{2}{|c|}{$\mathbf{L 3}$} & \multicolumn{2}{|c|}{ L4 } & \multicolumn{2}{|c|}{ LT } \\
\hline & & $\begin{array}{l}\text { Cros- } \\
\text { corr }\end{array}$ & $\begin{array}{c}\text { Lag } \\
\text { (s) }\end{array}$ & $\begin{array}{l}\text { Cros- } \\
\text { corr }\end{array}$ & $\begin{array}{l}\text { Lag } \\
\text { (s) }\end{array}$ & $\begin{array}{c}\text { Cros- } \\
\text { corr }\end{array}$ & $\begin{array}{c}\text { Lag } \\
\text { (s) }\end{array}$ & $\begin{array}{l}\text { Cros- } \\
\text { corr }\end{array}$ & $\begin{array}{l}\text { Lag } \\
(\mathrm{s})\end{array}$ & $\begin{array}{c}\text { Cros- } \\
\text { corr }\end{array}$ & $\begin{array}{l}\text { Lag } \\
\text { (s) }\end{array}$ & $\begin{array}{l}\text { Cros- } \\
\text { corr }\end{array}$ & $\begin{array}{l}\text { Lag } \\
\text { (s) }\end{array}$ \\
\hline \multirow[t]{2}{*}{1} & $P_{\text {mouth }}$ f-EDR & 0.91 & 0.98 & 0.88 & 0.78 & 0.89 & 0.67 & 0.83 & 0.67 & 0.86 & 0.65 & 0.71 & 0.68 \\
\hline & $P_{\text {mouth }}$ f-EMGDR & 0.93 & 0.70 & 0.95 & 0.35 & 0.95 & 0.33 & 0.94 & 0.33 & 0.94 & 0.22 & 0.91 & 0.29 \\
\hline \multirow[t]{2}{*}{2} & $P_{\text {mouth }}$ f-EDR & 0.91 & 0.99 & 0.88 & 0.77 & 0.90 & 0.68 & 0.90 & 0.66 & 0.83 & 0.66 & 0.72 & 0.69 \\
\hline & $P_{\text {mouth }}$ f-EMGDR & 0.91 & 0.65 & 0.93 & 0.28 & 0.95 & 0.21 & 0.93 & 0.16 & 0.95 & 0.02 & 0.87 & 0.15 \\
\hline
\end{tabular}




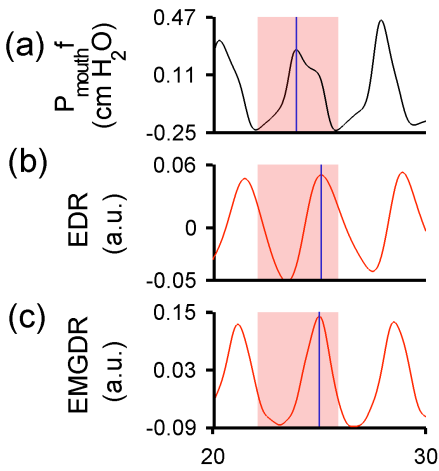

$t(s)$
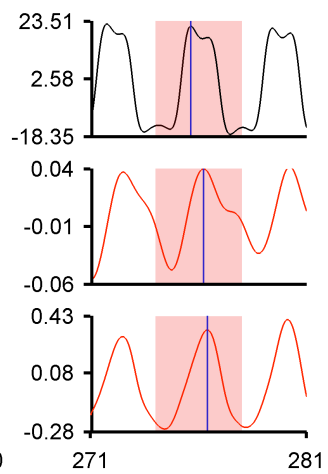

$t(s)$

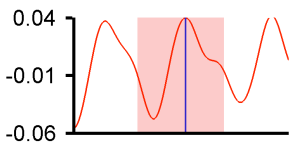

Figure 3. (a) $\mathrm{P}_{\text {mouth }} \mathrm{f}$ (b) EDR signal and (c) EMGDR signal using fSampEn at quiet breathing (left column) and $33 \mathrm{cmH}_{2} \mathrm{O}$ (right column). Vertical lines indicate the maximum of the chosen respiratory cycle highlighted by vertical light red rectangles.

High cross-correlation values are observed at each level of inspiratory load, being higher between $\mathrm{P}_{\text {mouth }} \mathrm{f}$ and EMGDR $(\geq 0.91)$ than between $\mathrm{P}_{\text {mouth }} f$ and EDR results $(\geq 0.83)$. When the set of all inspiratory loads were evaluated, the crosscorrelation value was higher also between $\mathrm{P}_{\text {mouth }} \mathrm{f}$ and EMGDR $(\geq 0.87)$ than between $P_{\text {mouth }} f$ and EDR $(\geq 0.71)$. In this case the difference is greater because, unlike $\mathrm{P}_{\text {mouth }} \mathrm{f}$ and EMGDR signals amplitude, the amplitude of the EDR do not increase with the increase of the inspiratory load (as can be observed in Fig. 2 and 3). The lags between $P_{\text {mouth }} f$ vs EMGDR were lower $(\leq 0.70 \mathrm{~s})$ than between $\mathrm{P}_{\text {mouth }} \mathrm{f}$ vs EDR results $(\leq 0.99 \mathrm{~s})$. The lag value was also lower between $\mathrm{P}_{\text {mouth }} \mathrm{f}$ vs EMGDR ( $\left.\leq 0.29 \mathrm{~s}\right)$ than between $\mathrm{P}_{\text {mouth }} \mathrm{f}$ vs EDR ( $\leq$ $0.69 \mathrm{~s}$ ), when the set of all inspiratory loads were evaluated. The respiratory rate values of the $P_{\text {mouth }} f$, EDR and EMGDR signals calculated at each level of inspiratory load are given in Table II.

It can be observed that there are slight differences in the results obtained through the EDR and EMGDR signals in comparison to the $\mathrm{P}_{\text {mouth }} \mathrm{f}$ signal. This implies that it is possible to derive the respiratory rate from the EMGDR signal.

\section{Discussion AND CONCLUSION}

The present work introduces the use of the fSampEn method to obtain the respiration waveform from the respiratory muscle electrical activity with the intention to extract the respiratory rate. This study was conducted in healthy subjects performing an inspiratory load protocol, in which the increase of the $\mathrm{P}_{\text {mouth }}$ increases the inspiratory muscle activity. fSampEn has been found to be suitable for the non-invasive evaluation of the muscle activity contaminated with ECG and to obtain the EMGDR signal. Indeed, it has been observed that the EMGDR signal was successfully calculated in the whole set of inspiratory loads, that is, from the quiet breathing (without an imposed inspiratory load) to the $33 \mathrm{~cm} \mathrm{H}_{2} \mathrm{O}$ (maximum imposed inspiratory load). Furthermore, the performance of the EMGDR was higher in comparison to the EDR signal. Additionally, the estimation of the respiratory rate from the three signals $\left(\mathrm{P}_{\text {mouth }}, \mathrm{EDR}\right.$ and EMGDR) were similar, which suggest that the EMGDR can be used to extract useful
TABLE II. RESPIRATORY RATE AT DIFFERENT LEVELS OF INSPIRATORY LOAD

\begin{tabular}{|c|l|l|l|l|l|l|}
\hline Subject & & $\begin{array}{c}\text { QB } \\
(\mathbf{b p m})\end{array}$ & $\begin{array}{c}\text { L1 } \\
(\mathbf{b p m})\end{array}$ & $\begin{array}{c}\text { L2 } \\
(\mathbf{b p m})\end{array}$ & $\begin{array}{c}\mathbf{L 3} \\
(\mathbf{b p m})\end{array}$ & $\begin{array}{c}\mathbf{L 4} \\
(\mathbf{b p m})\end{array}$ \\
\hline \multirow{3}{*}{1} & $\mathbf{P}_{\text {mouth }} \mathbf{f}$ & 15.61 & 15.75 & 15.66 & 15.61 & 15.70 \\
\cline { 2 - 7 } & EDR & 15.66 & 15.70 & 15.66 & 15.70 & 15.70 \\
\cline { 2 - 7 } & EMGDR & 15.66 & 15.75 & 15.70 & 15.66 & 15.70 \\
\hline \multirow{3}{*}{2} & $\mathbf{P}_{\text {mouth }} \mathbf{f}$ & 15.70 & 15.66 & 15.56 & 15.66 & 15.66 \\
\cline { 2 - 7 } & EDR & 15.70 & 15.56 & 15.61 & 15.66 & 15.70 \\
\cline { 2 - 7 } & EMGDR & 15.70 & 15.61 & 15.56 & 15.61 & 15.70 \\
\hline \multicolumn{5}{|c|}{ QB: Quiet breathing (without inspiratory load). }
\end{tabular}

L1 to L4: inspiratory load corresponding to $9,17,25$ and $33 \mathrm{cmH}_{2} \mathrm{O}$.

bpm: breaths per minute.

information from the respiration. Further work should be addressed to extend the use of fSampEn in a large number of healthy subjects and in patients suffering from chronic diseases.

\section{REFERENCES}

[1] N. M. Punjabi, B. S. Caffo, J. L. Goodwin, D. J. Gottlieb, A. B. Newman, et al., "Sleep-disordered breathing and mortality: A prospective cohort study," PLoS Med., vol. 6, no. 8, p. e1000132, 2009.

[2] A. M. Yañez, D. Guerrero, R. Pérez De Alejo, F. Garcia-Rio, J. L. Alvarez-Sala, et al., "Monitoring breathing rate at home allows early identification of COPD exacerbations," Chest, vol. 142, no. 6, pp. 1524-1529, 2012.

[3] A. Garde, W. Karlen, J. M. Ansermino, and G. A. Dumont, "Estimating respiratory and heart rates from the correntropy spectral density of the photoplethysmogram," PLoS One, vol. 9, no. 1, p. e86427, Jan. 2014.

[4] G. B. Moody and R. G. Mark, "Derivation of respiratory signals from multi-lead ECGs," Comput. Cardiol., vol. 12, pp. 113-116, 1985.

[5] S. Babaeizadeh, S. H. Zhou, S. D. Pittman, and D. P. White, "Electrocardiogram-derived respiration in screening of sleepdisordered breathing," J. Electrocardiol., vol. 44, no. 6, pp. 700-706, 2011.

[6] G. B. Moody, R. G. Mark, M. a Bump, J. S. Weinstein, A. D. Berman, et al., "Clinical Validation of the ECG-Derived Respiration (EDR) Technique,” Comput. Cardiol., vol. 13, pp. 507-510, 1986.

[7] A. Konik, P. Dasari, J. M. Mukherjee, K. L. Johnson, E. Helfenbein, et al., "Respiratory tracking using EDR for list-mode binning in cardiac emission tomography: Comparison with MRI heart motion measurements," in Nuclear Science Symposium and Medical Imaging Conference (NSS/MIC), 2012, pp. 2131-2136.

[8] P. C. W. Pang, M. G. Pepper, and D. a. Ducker, "Monitoring respiratory activity in neonates using diaphragmatic electromyograph," Med. Biol. Eng. Comput., vol. 33, no. 3, pp. 385390, May 1995.

[9] J. S. Richman and J. R. Moorman, "Physiological time-series analysis using approximate entropy and sample entropy," Am J Physiol Hear. Circ Physiol, vol. 278, no. 6, pp. H2030-H2049, 2000.

[10] L. Sarlabous, A. Torres, J. a Fiz, and R. Jané, "Evidence towards Improved Estimation of Respiratory Muscle Effort from Diaphragm Mechanomyographic Signals with Cardiac Vibration Interference Using Sample Entropy with Fixed Tolerance Values," PLoS One, vol. 9, no. 2, p. e88902, Jan. 2014.

[11] L. Estrada, A. Torres, L. Sarlabous, and R. Jane, "Improvement in Neural Respiratory Drive Estimation from Diaphragm Electromyographic Signals using Fixed Sample Entropy," IEEE J. Biomed. Heal. Informatics, vol. 2194, no. c, pp. 1-1, 2015. DOI: 10.1109/JBHI.2015.2398934.

[12] C. J. Jolley, Y. M. Luo, J. Steier, C. Reilly, J. Seymour, et al., "Neural respiratory drive in healthy subjects and in COPD," Eur. Respir. J., vol. 33 , no. 2, pp. 289-297, 2009. 